ORIGINAL ARTICLE

\title{
Neonatal Hyperbilirubinemia treatment by Locally Made Low-Cost Phototherapy Units
}

\author{
Netsanet Workneh Gidi ${ }^{1,2 *}$, Matthias Siebeck ${ }^{3}$
}

\section{OPEN ACCESS}

Citation: Netsanet Workneh Gidi, Matthias Siebeck. Neonatal Hyperbilirubinemia treatment by Locally Made Low-Cost

Phototherapy Units. Ethiop J Health

Sci.

doi:http://dx.doi.org/

10.4314/ejhs.v31i1.7

Received: October 9, 2019

Accepted: March 5, 2020

Published: January 1, 2021

Copyright: (C) 2020 Netsanet Workneh Gidi, et al. This is an open access article distributed under the terms of the Creative Commons Attribution License, which permits unrestricted use, distribution, and reproduction in any medium, provided the original author and source are credited.

Funding:This study was supported by Grand Challenges Canada

Competing Interests:The authors declare that this manuscript was approved by all authors in its form and that no competing interest exists. Affiliation and Correspondence:

${ }^{1}$ Jimma University, Jimma, Ethiopia.

${ }^{2}$ CIHLMU Center for International Health, Medical Center of the University of Munich (LMU), Germany

${ }^{3}$ Medical Center of the University of Munich (LMU), Germany

*Email: konetsanet@gmail.com

\begin{abstract}
BACKGROUND: Hyperbilirubinemia is a very common finding in neonates and may occasionally cause severe morbidity and even mortality. Severe hyperbilirubinemia is typically treated, either with phototherapy or exchange transfusions. This study assessed the effectiveness of a locally manufactured phototherapy device for reducing serum bilirubin in neonates with severe hyperbilirubinemia.

METHODS: Retrospective chart review was carried out to assess the outcome of 32 infants who were treated for neonatal hyperbilirubinemia at Jimma Medical Center (JMC) from May, 2017 to April, 2018. RESULTS: Out of 75 charts reviewed, only 32 had subsequent bilirubin level determination, 18(56.3\%) of them were males. The age at which jaundice was noticed and confirmed with plasma bilirubin level was $4 \pm 2.7$ days (mean+SD). Sepsis was thought to be the cause of hyperbilirubinemia in 13(40.5\%) of the cases, while hemolysis from $A B O$ incompatibility or $\mathrm{RH}$ incompatibility contributed in 5(15.6\%) and 3(9.4) of the infants respectively. The mean (minimum, maximum) level of baseline TSB was $21.4(14,55) \mathrm{mg} / \mathrm{dL}$. Five infants $(15.6 \%)$ had exchange transfusions because of extreme hyperbilirubinemia. The duration of phototherapy and decline in TSB were $5.34 \pm 2.8$ days and $2.2+1.5 \mathrm{mg} / \mathrm{dl} / \mathrm{day}$ (mean \pm SD) respectively. The levels of TSB before and at the end of phototherapy were significantly different $(p<0.001)$.

CONCLUSION: Acceptable reduction of TSB was achieved by using locally manufactured PT devices. Benefits included better accessibility and lower price and maintenance costs. High mean baseline TSB was observed, and duration of phototherapy is prolonged which could indicate late diagnosis compared to similar studies.
\end{abstract}

\section{INTRODUCTION}

Acute bilirubin encephalopathy (ABE) or kernicterus is a feared complication arising from untreated severe neonatal hyperbilirubinemia and a leading cause of preventable brain damage $(1,2)$. This unacceptably high global burden of severe neonatal hyperbilirubinemia and associated brain damage is a preventable and/or treatable disorder that requires implementation of existing 
technology to reduce the associated morbidity and mortality (3). Neonatal hyperbilirubinemia is a very common and mostly benign transitional condition that can be seen during the first few days of life in $60 \%-80 \%$ of newborns worldwide (4). Further studies are highly needed to understand the profile of infants with or at risk of severe hyperbilirubinemia in most lowincome and middle-income countries (LMICs) (5).

Although the term "severe hyperbilirubinemia" is not strictly defined, the following elements have been proposed: a newborn who develops visible jaundice within the first 24 hours of life, when total serum bilirubin (TSB) levels increase $>5 \mathrm{mg} / \mathrm{dl} /$ day, when peak levels are higher than the expected normal range (i.e. $>95^{\text {th }}$ percentile), when clinically visible jaundice persists for more than 2 weeks after birth, and when there is evidence of conjugated hyperbilirubinemia (6). Kernicterus Spectrum Disorder (KSD) is characterized by long-term neurodevelopmental abnormalities, including dyskinetic (choreoathetoid) cerebral palsy, paresis of upward gaze, developmental delay, cognitive impairment, disordered executive function, language processing disorders, as well as behavioral and psychiatric disorders (7).

Common causes that can contribute to severe neonatal jaundice (NJ) include hemolysis due to $\mathrm{Rh}$ factor or $\mathrm{ABO}$ blood group incompatibility, glucose-6-phosphate dehydrogenase (G6PD) deficiency and other hemolytic disorders, as well as breastfeeding or breast milk, premature birth, infection, cephalohematoma or other circumscribed hemorrhages, and asphyxia $(8,9)$.Timely identification of risk factors that predispose to severe hyperbilirubinemia and neurotoxicity is important in order to be able to start treatment on time and thus decrease mortality and morbidity (3).

Phototherapy is the preferred method of treatment for severe neonatal unconjugated hyperbilirubinemia regardless of its etiology. Phototherapy works most effectively at blue light wavelengths $(450 \pm 20 \mathrm{~nm})$, preferably placed close to the infant $(30 \mathrm{~cm}$ distance or less), and with as much of the infant's body surface area exposed as possible, in order to maximize spectral power $(10,11)$. Guidelines for phototherapy exist in several formats and typically relate TSB thresholds to the infant's maturity (either represented by gestational age or birthweight), postnatal age (in hours), and presence of factors/conditions that increase the risk for bilirubin neurotoxicity(12). Such risk factors may include isoimmune hemolytic disease, glucose-6-phosphate dehydrogenase deficiency, asphyxia, lethargy, temperature instability, sepsis, acidosis, and hypoalbuminemia $(12,16)$. Exchange transfusion (ET) is considered the most effective measure to rapidly lower the bilirubin level to prevent acute bilirubin encephalopathy and progression to KSD. However, unlike phototherapy, it is invasive and associated with complications of blood transfusion and the procedure $(3,13-15)$.

The problem of severe $\mathrm{NJ}$ and gaps in implementing effective strategies for identification, prevention, and treatment are still significant in several LMICs. Standard phototherapy machines are expensive and not readily available in the market in many LMICs. Thus, there is a great demand for affordable alternative devices in these settings $(4,19,20)$. The aim of this study was to assess how well locally made low cost phototherapy devices worked in the treatment of NJ.

\section{MATERIALS AND METHODS}

The study was conducted in the neonatal intensive care unit (NICU) at JMC, Jimma, Ethiopia. JMC is one of the tertiary hospitals in Ethiopia, located in the Southwest part of the country, with over 600 beds capacity. The pediatric unit of JMC has a NICU with over 25 beds. Neonates admitted to the unit are either referred from the surrounding health centers or the JMC delivery room. JMC NICU has been using locally made phototherapy units since May 2017, as there was no functional phototherapy unit available at the hospital. Several expensive machines were out of use at the same time, due to lack of maintenance and lack of availability of spare parts.

This was a retrospective cross-sectional study. Neonates (age $<28$ days) admitted with the diagnosis of hyperbilirubinemia from May 2017 to April 2018 at Jimma Medical Center 
were the study population. Neonates diagnosed with hyperbilirubinemia were identified from neonatal ward logbook, and patient charts were retrieved. The data was collected using structured chart review guide developed for this study. Data was collected by physicians from neonatal ward logbook and patient charts using structured chart review guide. The data collectors were trained for the purpose of the study and study procedures prior to commencement of data collection. Patient and mothers' characteristics, ABO blood group and $\mathrm{RhD}$ antigen typing of mother and child, other possible causes of hyperbilirubinemia, levels of serum bilirubin, duration of therapy, and outcome were reviewed using a structured chart review guide. Data was checked for completeness, cleaned, and entered into SPSS version 22 for analysis. Descriptive statistics was used where appropriate. Paired sample $T$ test was done to assess for statistically significant reduction of TSB after treatment with phototherapy. The study was conducted after ethical approval was obtained from Jimma
University IRB, reference number: IHRPGD/456/2018.

Simbona Africa Healthcare R \& D, an organization founded by Mr. Habtamu Abafogi, provided the locally made phototherapy units which the NICU has been using for about a year. The components used to construct phototherapy units comply with medically recommended specifications (Table 1). The machine is easy to install and maintain, lamps can be replaced easily, for LED's the whole board can be replaced by technicians on site, it can be disinfected by available disinfectants and can operate at $25{ }^{\circ} \mathrm{C}-50{ }^{\circ} \mathrm{C}$. Technical (engineering) evaluation of the phototherapy units was done by Jimma University Biomedical Engineering Centre. Irradiance level was $18 \mu \mathrm{W} / \mathrm{cm} 2 / \mathrm{nm}$ as measured by a radiometer. Electronics board safety, wavelength, and irradiance level were confirmed to be in accordance with international standard guidelines ISO 900:IEC 60601-52 for neonatal phototherapy units (reference number: BME-JUMC-182-10).

Table 1: Specifications of basic technology used to construct phototherapy units by Simbona Africa Healthcare R\&D, Ethiopia.

\begin{tabular}{|c|c|c|c|c|}
\hline No & Components & Specifications & Brand & $\begin{array}{l}\text { Companies currently } \\
\text { using the components }\end{array}$ \\
\hline 1 & Blue LED & $\begin{array}{ll}\text { - } & \text { Wave length (450- } \\
& \text { 470nm) } \\
\text { - } & \text { SMD Blue LEDs } \\
\text { - } & \text { DIP Blue LEDs } \\
\text { - } & \text { Life time:50000hrs }\end{array}$ & CE-Market & $\begin{array}{ll}\text { - } & \text { Fanem Phototherapy } \\
\text { - } & \text { Natus PT }\end{array}$ \\
\hline 2 & CFL & $\begin{array}{ll}- & \text { Wavelength } 450 \mathrm{~nm} \\
- & 10,000 \text { hours }\end{array}$ & CE-Market & $\begin{array}{ll}\text { - } & \text { Olympic } \\
\text { - } & \text { Sylvania } \\
\text { - } & \text { neoBlue } \\
\text { - } & \text { Philips and other }\end{array}$ \\
\hline 3 & Timer & 6 digit counter & CE-Market & Philips phototherapy \\
\hline
\end{tabular}

\section{RESULTS}

Four locally made phototherapy units were in use: two CFL and two LED. There were 87 infants diagnosed with neonatal jaundice requiring treatment, accounting for $8.5 \%$ of total NICU admission in the year. Out of 75 charts retrieved (12 charts could not be accessed), only 32 had follow-up TSB determinations. The rest were either treated on clinical basis without measuring TSB or did not have hyperbilirubinemia in the range phototherapy is indicated (Figure 1). The report thus covers the 
outcome of 32 infants who had adequate TSB determinations both before and after treatment with phototherapy.

The majority of infants $19(59.4 \%)$ were born in JUMC; while 11 (34.4\%) were referred from the surrounding health centers and hospitals, the remaining $2(6.3 \%)$ were born at home. All of the mothers had antenatal care, of these 4 were identified with antenatal hemorrhage, preeclampsia, $\mathrm{Rh}$ - negative mother, and thyrotoxicosis. The mode of delivery was spontaneous vertex delivery in $27(62.8 \%)$ of the cases, while $10(31.3 \%)$ and $2(6.3 \%)$ were born by caesarian section and instrumental delivery, respectively.

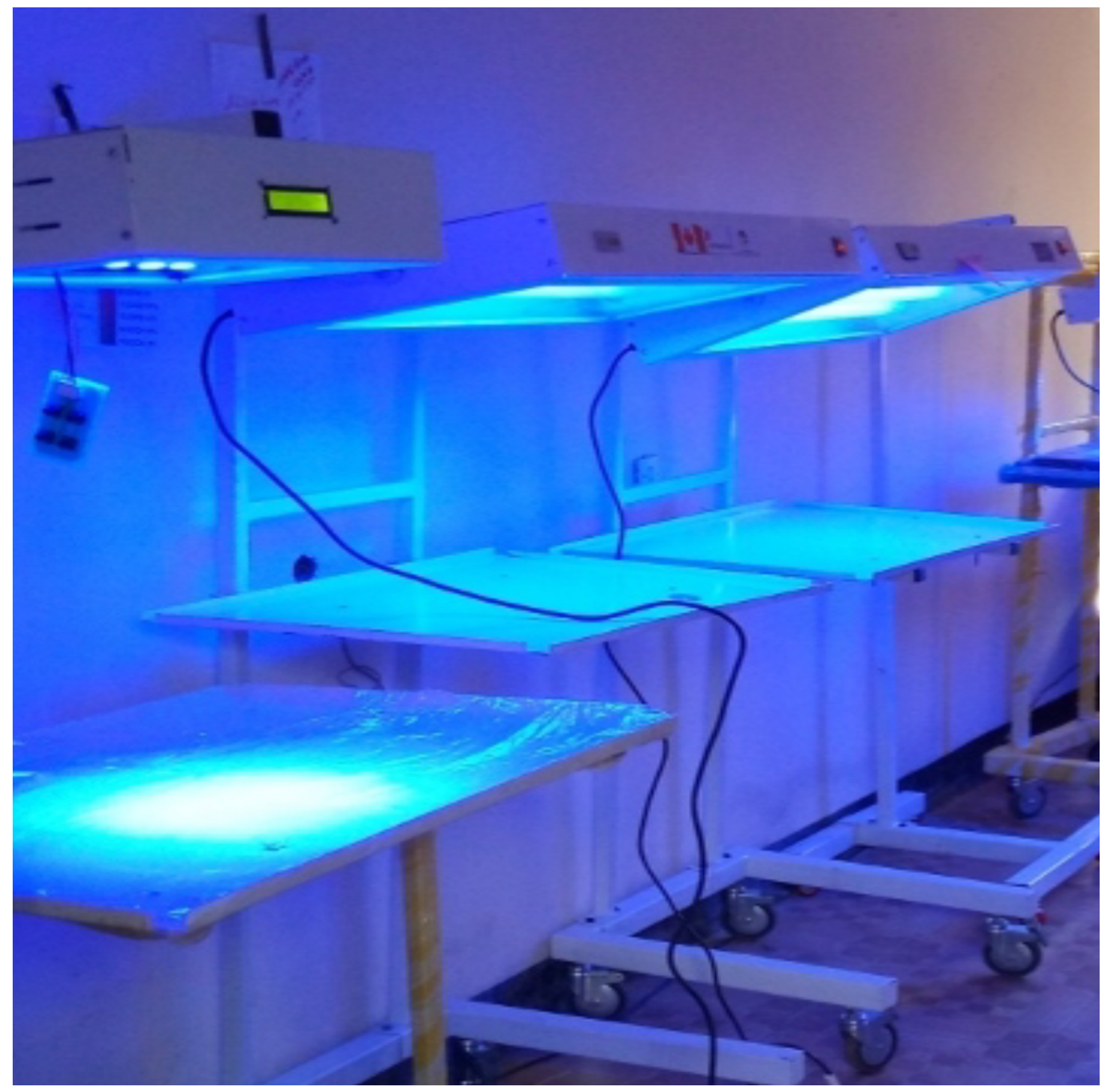

Figure 1:- Phototherapy devices developed by Simbona Africa Healthcare R\&D, Ethiopia. 
Eighteen $(56.3 \%)$ infants were male. The age at which jaundice was clinically noticed and confirmed with a TSB level was day $4 \pm 2.7$ (mean $\pm \mathrm{SD}$ ). As routine screening is not included in the guidelines for the unit, all the infants were tested following clinical suspicion of severe jaundice. The birth weight and GA of the infants were $2825 \pm 817 \mathrm{G}$ and $36.8 \pm 2.6$ weeks respectively (mean $\pm \mathrm{SD}$ ). Sepsis was identified as the main cause of hyperbilirubinemia in $13(40.5 \%)$ of the cases, while hemolysis from $\mathrm{ABO}$ incompatibility and $\mathrm{Rh}$ incompatibility were seen in 5(15.6\%) of the infants. Only one of the infants had clinical signs of acute bilirubin encephalopathy on admission. The level of TSB and HCT were $21.4(14,55)$ and $44.7(28,62)$ respectively (mean (range) (Table 2).

Table 2: Perinatal data of infants with pathologic jaundice

\begin{tabular}{ll}
\hline Variables & Values \\
\hline Males No. (\%) & $18(56.3)$ \\
GA weeks, mean (SD) & $36.8(2.6)$ \\
Birth weight G, mean (SD) & $2825(817)$ \\
Parity of the mother, mean (SD) & $2.7(1.8)$ \\
Place of delivery, No. (\%) & \\
JUMC & $19(59.4)$ \\
Health centers and other hospitals & $11(34.4)$ \\
Home & $2(6.3)$ \\
Mode of delivery, n(\%) & \\
Spontaneous vaginal & $20(62.5)$ \\
Caesarean section & $10(31.3)$ \\
Instrumental & $2(6.3)$ \\
Age at diagnosis of hyperbilirubinemia in days, mean (SD) & \\
Causes of hyperbilirubinemia No. (\%) & $4(2.7)$ \\
Sepsis & \\
ABO incompatibility & $13(40.5)$ \\
Rn incompatibility & $5(15.6)$ \\
Subgaleal hemorrhage & $3(9.4)$ \\
Breast feeding jaundice & $3(9.4)$ \\
Hypothyroidism & $2(6.3)$ \\
Unknown cause & $1(3.1)$ \\
TSB (g/dL) at start of phototherapy, mean (range) & $5(15.6)$ \\
Hematocrit, mean (range) & $21.4(14,55)$ \\
\hline
\end{tabular}

Five of the babies had TSB value above the level at which exchange transfusion is indicated. However, only three of them got exchange transfusion while the other two were treated with phototherapy. The total duration of phototherapy and average decline in TSB level were 5.34 \pm 2.8 days and $2.2 \pm 1.5 \mathrm{mg} / \mathrm{dl} /$ day (mean $\pm \mathrm{SD})$. The mean reduction of TSB after exchange transfusion was $9.9 \mathrm{mg} / \mathrm{dL} /$ procedure. Paired sample test analysis showed significant difference in average levels of TSB before and at after phototherapy $\left(\mathrm{t}_{31}=6.2, \mathrm{p}<0.001\right)$. The majority of infants, $31(96.9 \%)$, were discharged with improved condition while one of the infant's family left against medical advice (Table 3). 
Table 3: Treatment and outcome of hyperbilirubinemia $(\mathrm{No}=32)$.

\begin{tabular}{ll}
\hline Variables & Values \\
\hline TSB>Exchange transfusion level, No. (\%) & $5(15.6)$ \\
Total duration of phototherapy in days, mean (SD) & $5.34(2.8)$ \\
Average decline in TSB in mg/dl/day, mean (SD) & $2.2(1.5)$ \\
Average decline in TSB in mg/dl/procedure, mean (SD) & $9.9(3.3)$ \\
Outcome at discharge & \\
$\quad$ Survived & $31(96.9)$ \\
Left against medical advice & $1(3.1)$ \\
Death & $0(0)$ \\
\hline
\end{tabular}

\section{DISCUSSION}

Herein we show that locally made phototherapy units can be used effectively to treat neonatal hyperbilirubinemia. The lamps used in our devices were sourced locally, a significant advantage compared to imported units, for which spare parts and lamps may either not be available at all, or at significantly higher cost or long delays as far as delivery from the manufacturers (10). Although the basic concept and design of phototherapy devices is rather simple, technological sophistication in the newer generations of such devices means that the standard models on the market are too expensive for resource poor settings, with costs ranging from US\$1200 to 1500 , the manufacturing cost of the locally made phototherapy was about 400 US\$. Many devices currently in use in LMIC hospitals were donated from hospitals in developed countries, often not accompanied by adequate supplies of spare parts, and they are not often checked for proper functioning, including irradiance $(21,26)$. Because severe NJ continues to be an important health problem in LMICs (5), with the tragic sequelae of KSD contributing significantly to the burden of neurodevelopmental impairment in LMIC populations (4); many ways to increase the availability of treatment tools deserve to be explored $(10,14)$.

A variety of phototherapy devices are considered effective in decreasing TSB (21). The mean daily TSB decrement observed in this study $(2.1 \mathrm{mg} / \mathrm{dl})$ is comparable with the finding of others. Thus, Brown et al used daylight fluorescent tubes and reported a TSB decrease of $1.5 \mathrm{mg} / \mathrm{dL}$ during the first day of phototherapy
(22). However, our numbers are lower than those in a report of a study that compared the effectiveness of LED versus CFL, with average decreases of TSB levels of $4.1 \pm 0.48$ and $4.8 \pm$ $0.24 \mathrm{mg} / \mathrm{dL}$ respectively at the end of 24 hours (23).

The mean duration of phototherapy of 5.3 days in our study is longer than the findings of similar studies, average of 1-3 days which can be explained by late diagnosis requiring prolonged period to treat, lack of universal screening of newborns with TSB or cutaneous bilirubin, TSB being determined only when jaundice is clinically diagnosed. On the same line, the mean baseline TSB of the infants was greater than the levels reported by studies compared $(24,25)$. Generally, phototherapy is considered safe with minimal risk, and none of the infants treated with PT in this study had reports of significant complications (24).

Measuring the dose of phototherapy is required to insure the efficacy of the device (12). However, determining device irradiance is not widely practiced, both due to lack of irradiance meters (radiometers specific to the device as recommended by manufacturer) (12), and also to limited knowledge of its importance by health professionals. Lack of maintenance and unavailability of replacement parts are also major impediments to sustainable, effective PT units in LMICs $(21,26)$. The manufacturing cost of the present locally made CFL and LED phototherapy units is about one-fourth of the price of the standard models in the market, with the possibility of maintenance at the site in case of malfunctioning and irradiance checks, which makes it attractive given the high demand. 
The limitation of this study include retrospective data collection, and associated incomplete patient data. This forced us to exclude some neonates who had neonatal jaundice and received phototherapy. Some of the cases did not have adequate follow up bilirubin measurement recorded on their charts. For these reasons smaller number of neonates receiving phototherapy were included for analyis.

Acceptable decrement in TSB was achieved by using locally made phototherapy devices (CFL and LED). Additional benefits include better accessibility, lower cost and easier access to maintenance. We observed a high mean baseline TSB, and duration of phototherapy was prolonged, pointing to late diagnosis compared to similar studies. The cause of prolonged duration of therapy could be related to the fact that the phototherapy was conventional instead of intensive. However, understanding the exact reason why duration of therapy was prolonged requires further investigation. Adopting universal screening for hyperbilirubinemia with TSB or noninvasive cutaneous bilirubin determination could help to identify infants in need of therapy earlier.

\section{REFERENCES}

1. Babu TA, Bhat BV, Joseph NM. Neurobehavior of term neonates with neonatal hyperbilirubinemia. Journal of pediatric neurosciences 2013;8(1):11.

2. Hameed NN, Vilms R, Bhutani VK. Severe neonatal hyperbilirubinemia and adverse short-term consequences in Baghdad, Iraq. Neonatology 2011; 100(1):57-63.

3. Singla DA, Sharma S, Sharma M, Chaudhary S. Evaluation of risk factors for exchange range hyperbilirubinemia and neurotoxicity in neonates from hilly terrain of India. International Journal of Applied and Basic Medical Research 2017; 7(4):228.

4. Olusanya BO, Ogunlesi TA, Slusher TM. Why is kernicterus still a major cause of death and disability in low-income and middle-income countries?. Archives of disease in childhood 2014; 99(12):1117-21.

5. Olusanya BO, Osibanjo FB, Slusher TM. Risk factors for severe neonatal hyperbilirubinemia in low and middleincome countries: a systematic review and meta-analysis. PLoS One 2015; 10(2): 0117229.

6. Ullah S, Rahman $\mathrm{K}$, Hedayati $\mathrm{M}$. Hyperbilirubinemia in neonates: types, causes, clinical examinations, preventive measures and treatments: a narrative review article. Iranian journal of public health 2016; 45(5):558.

7. Wusthoff CJ, Loe IM. Impact of bilirubininduced neurologic dysfunction on neurodevelopmental outcomes. InSeminars in Fetal and Neonatal Medicine 2015; 20(1): 52-57

8. Boyd S. Treatment of physiological and pathological neonatal jaundice. Nursing times 2004; 100(13):40-3.

9. Huang MJ, Kua KE, Teng HC, Tang KS, Weng HW, Huang CS. Risk factors for severe hyperbilirubinemia in neonates. Pediatric research 2004 56(5):682.

10. Bhutani VK, Cline BK, Donaldson KM, Vreman HJ. The need to implement effective phototherapy in resourceconstrained settings. InSeminars in perinatology 2011; 35(3): 192-197.

11. Hansen TW. Therapeutic approaches to neonatal jaundice: an international survey. Clinical pediatrics 1996; 35(6):309-16.

12. Maisels MJ, McDonagh AF. Phototherapy for neonatal jaundice. New England Journal of Medicine 2008; 28;358(9):920-8.

13. Newman TB, Maisels MJ. Evaluation and treatment of jaundice in the term newborn: a kinder, gentler approach. Pediatrics 1992; 89(5):809-18.

14. Owa JA, Ogunlesi TA. Why we are still doing so many exchange blood transfusion for neonatal jaundice in Nigeria. World Journal of Pediatrics 2009; 5(1):51-5.

15. Bujandric N, Grujic J. Exchange transfusion for severe neonatal hyperbilirubinemia: 17 years' experience from Vojvodina, Serbia. Indian Journal of Hematology and Blood Transfusion 2016; 32(2):208-14.

16. Schwartz HP, Haberman BE, Ruddy RM. Hyperbilirubinemia: current guidelines and 
emerging therapies. Pediatric emergency care 2011; 27(9):884-9.

17. Olusanya BO, Imosemi DO, Emokpae AA. Differences between transcutaneous and serum bilirubin measurements in black African neonates. Pediatrics 2016; 138(3):e20160907.

18. Chimhini GL, Chimhuya S, Chikwasha V. Evaluation of transcutaneous bilirubinometer (DRAEGER JM 103) use in Zimbabwean newborn babies. Maternal health, neonatology and perinatology 2018; 4(1):1.

19. Slusher TM, Day LT, Ogundele T, Woolfield N, Owa JA. Filtered sunlight, solar powered phototherapy and other strategies for managing neonatal jaundice in low-resource settings. Early human development 2017; 114:11-5.

20. Slusher TM, Zipursky A, Bhutani VK. A global need for affordable neonatal jaundice technologies. InSeminars in perinatology 2011; 35(3):185-191.

21. Malkin R, Anand V. A novel phototherapy device. IEEE Engineering in Medicine and Biology Magazine 2010; 29(2):37-43.

22. Brown AK, Kim MH, Wu PY, Bryla DA. Efficacy of phototherapy in prevention and management of neonatal hyperbilirubinemia. Pediatrics 1985; 75(2):393-441.

23. Yilmaz A, Ozkiraz S, Akcan AB, Canpolat M. Low-cost home-use light-emitting-diode phototherapy as an alternative to conventional methods. Journal of tropical pediatrics 2015; 23;61(2):113-8.

24. Slater L, Brewer MF. Home versus hospital phototherapy for term infants with hyperbilirubinemia: a comparative study. Pediatrics 1984; 73(4):515-9.

25. Zainab K, Adlina S. Effectiveness of home versus hospital phototherapy for term infants with uncomplicated hyperbilirubinemia: a pilot study in Pahang, Malaysia. Med $J$ Malaysia 2004; 59(3):395-401.

26. Cline BK, Vreman HJ, Faber K, Lou H, Donaldson KM, Amuabunosi E, Ofovwe G, Bhutani VK, Olusanya BO, Slusher TM. Phototherapy device effectiveness in Nigeria: irradiance assessment and potential for improvement. Journal of tropical pediatrics 2013; 59(4):321-5. 